\title{
A INFLUÊNCIA DO PULSO DE INUNDAÇÃO NA ECOLIMNOLOGIA DE BAÍAS PANTANEIRAS: UM ESTUDO NA DINÂMICA DE INVERTEBRADOS AQUÁTICOS
}

\author{
THE FLOOD PULSE INFLUENCE IN \\ ECOLIMNOLOGICAL PANTANAL LAKES: A STUDY TO \\ UNDERSTAND THE BIODIVERSITY DYNAMICS OF \\ AQUATIC INVERTEBRATES
}

\section{Ernandes Sobreira Oliveira Junior ${ }^{1^{*}}$; Cristina Márcia de Menezes Butakka ${ }^{2}$ Carolina Joana da Silva ${ }^{2}$; Claumir Cesar Muniz $^{2}$}

${ }^{1}$ UNEMAT - Universidade do Estado de Mato Grosso, Av. Tancredo Neves, 1095 Cavalhada II, 78200-000 - Cáceres, MG. E-mail*ernandes.biodagua@yahoo.com.br

${ }^{2}$ UNEMAT - Universidade do Estado de Mato Grosso - Centro de Limnologia Biodiversidade e Etnobiologia do Pantanal - CELBE - Cidade Universitária - Campus Universitário de Cáceres, 78200-000 - Cáceres, MG

\begin{abstract}
RESUMO
Este estudo se propôs relacionar as variáveis ambientais com a biota presente em duas baías do Pantanal Matogrossense, próximas à cidade de Cáceres-MT. Foram coletados dados de variáveis bióticas e abióticas da água em seis pontos de amostragem durante um ano de pesquisa, compreendendo os quatro períodos hidrológicos do Pantanal. Foi encontrada rica fauna de invertebrados aquáticos associados à macrófitas, tendo a Classe Insecta maior representatividade. As variáveis oxigênio dissolvido, transparência e profundidade apresentaram diferenças estatisticamente significativas entre os períodos hidrológicos. Os grupos Nematoda, Ostracoda, Decapoda, Chironomidae, Dityscidae e Copepoda estiveram significativamente correlacionados com as variáveis estudadas. Estes resultados podem indicar que as variáveis limnológicas exerceram influência na comunidade de invertebrados aquáticos e que modificações na qualidade da água podem resultar em uma alteração na microbiota, a qual exerce papel fundamental no fluxo de matéria e ciclagem de nutrientes dentro de um ecossistema aquático.
\end{abstract}

Palavras-chave: Variáveis limnológicas. Invertebrados bentônicos. Pantanal Matogrossense.

\footnotetext{
ABSTRACT

This study aimed to compare the environmental variables with this biota in two bays in the Pantanal near the city of Cáceres-MT. Data of biotic and abiotic variables of the water sampling were analyzed on six sampling points for one year survey comprising four Pantanal hydrological periods. Rich invertebrate fauna associated with aquatic macrophytes were found; with the Class Insecta is
} 
that with greater representativeness. There were significant differences between periods for hydrological variables dissolved oxygen, transparency and depth. The most significant correlations occurred between groups Nematoda, Ostracoda, Decapoda, Chironomidae, Copepoda and Dityscidae with dissolved oxygen, conductivity and depth. These data may indicate that the limnological variables influence in the aquatic invertebrates community and changes in water quality may result in a change in the microbiota, which is plays a fundamental role in the flow of matter and nutrient cycling within an ecosystem.

Keywords: Limnological variables. Benthic invertebrates. Pantanal.

\section{INTRODUÇÃO}

O Pantanal é um sistema frágil com formação geológica recente, possui $140.000 \mathrm{~km}^{2}$ de área inundável e tem como principal tributário o rio Paraguai, cujo regime hidrológico é responsável pela composição da biota terrestre e aquática (HARRIS et al., 2005).

O pulso de inundação, o "vai e vem” das águas, segundo Junk e Da Silva (1999), representa o principal processo ecológico em que as áreas permanentemente inundadas (rios, "baías") ou permanentemente secas ("cordilheiras") abrigam diferentes comunidades e uma rica biota capaz de resistir a essas fortes mudanças.

As baías pantaneiras, em sua grande maioria, possuem características semelhantes com baixas profundidades e funcionam como um receptáculo de material alóctone, o que as tornam suscetíveis às alterações de temperatura, oxigênio dissolvido, matéria orgânica e $\mathrm{pH}$, e exercem influência na comunidade aquática local (MUNIZ, 2005). Para Zillmer et al. (2007), essas características representam, além de condutividade e vazão, as principais para avaliação da alteração de um corpo hídrico.

As características físicas, químicas e biológicas de um ecossistema aquático são resultados da integração de vários fatores ambientais, sejam eles antrópicos, ou até mesmo os tipos de vegetação, solo, ou, no caso da planície pantaneira, a dinâmica do pulso de inundação (DA SILVA e PINTO-SILVA, 1989).

O estudo dos invertebrados aquáticos favorece o entendimento da estrutura trófica dos ecossistemas aquáticos, oferecendo subsídios para melhor compreensão desses sistemas, sua conservação, controle de poluição e de doenças, sua utilização em piscicultura e outras atividades produtivas, além de seu uso como organismos bioindicadores (GOULART e CALLISTO, 2003; FERREIRA et al., 2011).

Nos ambientes lênticos, os invertebrados desempenham fundamental papel, participando do processo da decomposição da matéria orgânica, reduzindo o tamanho das partículas, e são importantes na cadeia alimentar (ESTEVES, 1998), servindo de alimento para diversas espécies de peixes de água doce (RUSSO et al., 2002).

Informações das alterações hidrológicas e dos invertebrados são valiosas para o entendimento da estrutura e funcionamento das comunidades límnicas, e para registros dos processos ecológicos decorrentes das mudanças ocasionadas pelas variações hidrológicas. Este estudo poderá auxiliar no entendimento das relações dos organismos aquáticos (macrófitas/invertebrados) e servirá de base para futuros estudos de bioindicadores ambientais.

Esta pesquisa teve como objetivo analisar o efeito do pulso de inundação sobre a estrutura das comunidades de invertebrados associados às macrófitas aquáticas de dois subsistemas pantaneiros, e relacionar com as variáveis limnológicas no sistema. Os padrões de densidade numérica, diversidade, riqueza e uniformidade de larvas de Chironomidae são importantes na análise da estrutura da fauna associada. 


\section{MATERIAL E MÉTODOS}

O trabalho foi realizado na baía Caiçara, lagoa parental do rio Paraguai, localizada próximo à cidade de Cáceres, com de aproximadamente 20 hectares no período de estiagem, podendo dobrar de tamanho. Está situada à margem direita do rio Paraguai, entre as coordenadas $16^{\circ} 06^{\prime} 43,09^{\prime}$ S, $57^{\circ} 45^{\prime} 19,47^{\prime \prime} \mathrm{W}$, e $16^{\circ} 05^{\prime} 03,76^{\prime}$ ' S, $57^{\circ} 45^{\prime} 19,50^{\prime \prime} \mathrm{W}$, e comporta um sistema com duas porções: baía Caiçara Superior (BCS), subsistema que não recebe influxo de água do rio Paraguai no período de estiagem, e um subsistema inferior (BCI), que se mantém conectado com a calha do rio Paraguai em todos os períodos hidrológicos do pulso de inundação (Figura 1).
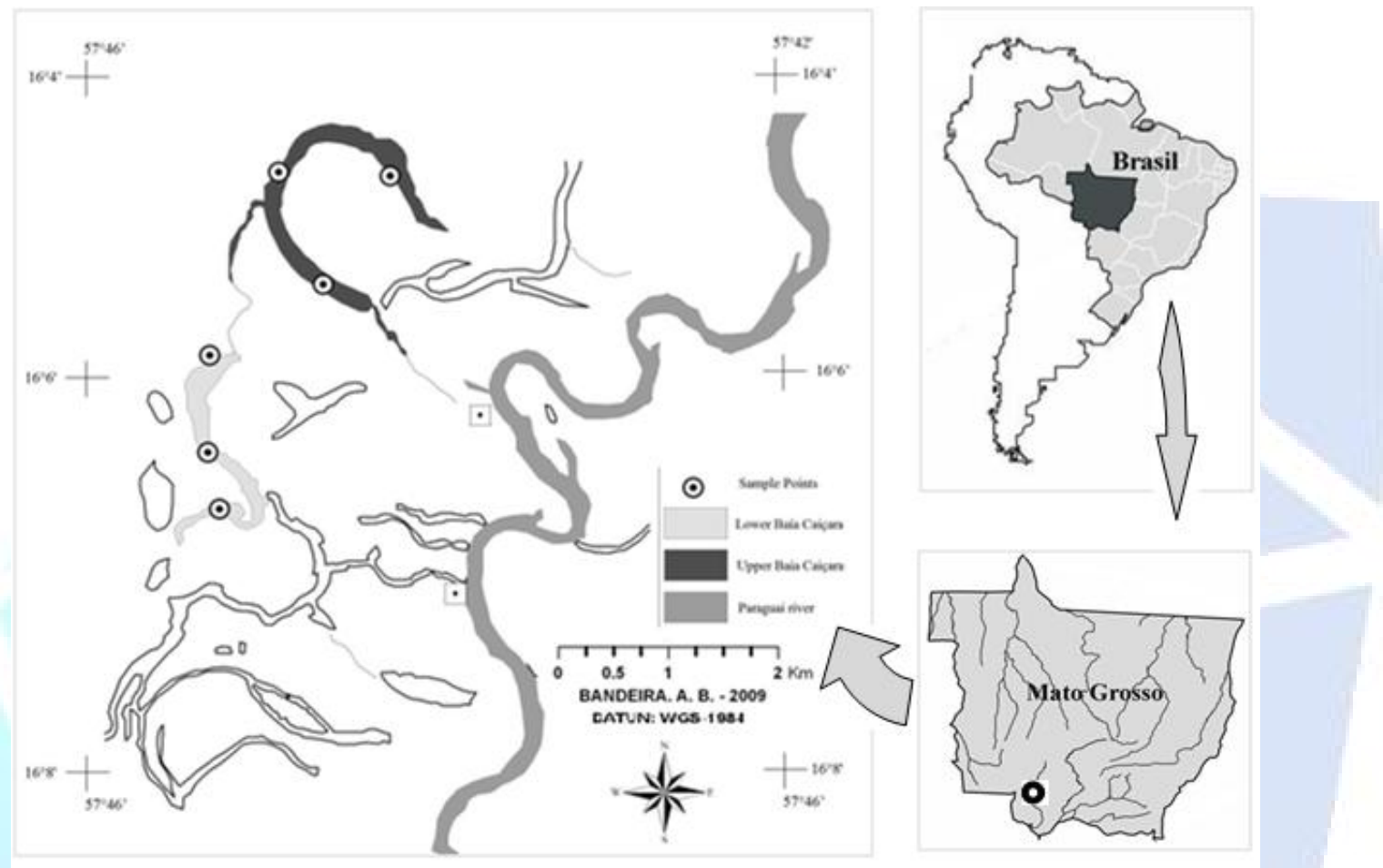

Figura 1. Localização dos pontos de amostragem na baía Caiçara (Upper Baía Caiçara - Baía Caiçara Superior $=$ BCS e Lower Baía Caiçara - Baía Caiçara Inferior = BCI) no Pantanal Matogrossense.

As amostras de invertebrados foram tomadas bimensalmente em seis pontos, sendo três na baía superior e três na inferior, com uma rede em formato de " $\mathrm{D}$ " e malha de $50 \mu \mathrm{m}$ em bancos de macrófitas aquáticas presentes na zona litorânea das baías durante um ano (agosto de 2006 a maio de 2007), compreendendo os quatro períodos hidrológicos do pulso de inundação (Estiagem, Enchente, Cheia, Vazante).

As variáveis limnológicas analisadas na pesquisa foram: profundidade da coluna de água $(\mathrm{cm})$, temperatura da água $\left({ }^{\circ} \mathrm{C}\right)$, condutividade $(\mu \mathrm{S} / \mathrm{cm})$, transparência $(\mathrm{cm})$, oxigênio dissolvido (mg/l) e potencial Hidrogeniônico $(\mathrm{pH})$.

Os invertebrados foram retirados das raízes das macrófitas aquáticas após sua lavagem, sendo triados ao nível de grandes grupos taxonômicos sob microscópio estereoscópico com a chave de Merritt et al. (2008). Para a identificação de grupos específicos foram utilizadas as chaves de Coffman e Ferrington Jr. (1996).

Para verificar as diferenças dos valores médios das variáveis limnológicas entre os períodos de amostragem e entre os subsistemas foi utilizada uma análise de variância (ANOVA). Através do Coeficiente de Correlação de Spearman $\left(r_{s}\right)$ não paramétrico, as associações foram geradas entre os 
dados bióticos e abióticos por meio do pacote estatístico XLStat (2003), com valores significativos para $\alpha=0,05$.

\section{RESULTADOS E DISCUSSÃO}

Entre os meses de agosto e setembro de 2006, foram registrados os níveis mais baixos do rio Paraguai, período caracterizado como estiagem. Além disso, a Baía Caiçara Superior (BCS) esteve totalmente desconectada do rio e da Baía Caiçara Inferior (BCI), não recebendo qualquer influxo de água. Nos meses de outubro e dezembro pôde ser observado o início da conexão entre BCS com o rio e com a BCI, identificando como o período de enchente, com o aumento da precipitação. O nível máximo do rio ocorreu nos mês de março de 2007, caracterizando o período de cheia. As águas começaram a baixar no começo do mês de abril, marcado como o período inicial de vazante. Os resultados limnológicos dos subsistemas BCS e BCI nos períodos estudados estão apresentados na Tabela 1.

Tabela 1 - Valores médios \pm desvios padrões das variáveis limnológicas dos subsistemas da baía Caiçara para os períodos de amostragem, entre agosto de 2006 e maio de 2007. BCS - baía Caiçara Superior; BCI baía Caiçara Inferior; ES - estiagem; EC - enchente; CH - cheia; VA - vazante; OD - oxigênio dissolvido; T temperatura da água; $\mathrm{Ce}$ - condutividade elétrica da água; $\mathrm{Tr}$ - transparência; $\mathrm{P}$ - profundidade.

\begin{tabular}{|c|c|c|c|c|c|c|c|c|c|c|c|c|c|}
\hline & & $\begin{array}{c}\text { OD } \\
(\mathrm{mg} / \mathrm{l})\end{array}$ & & $\begin{array}{c}\mathbf{T} \\
\left({ }^{\circ} \mathbf{C}\right)\end{array}$ & & $\begin{array}{c}\mathrm{Ce} \\
(\mathrm{S} / \mathrm{cm})\end{array}$ & & pH & & $\begin{array}{c}\text { Tr } \\
(\mathrm{cm})\end{array}$ & & $\begin{array}{c}\mathbf{P} \\
(\mathbf{c m})\end{array}$ & 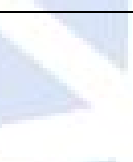 \\
\hline \multirow{4}{*}{ B } & ES & 4,69 & $\pm 0,67$ & 22,53 & $\pm 2,40$ & 49,70 & $\pm 0,19$ & 7,13 & $\pm 0,26$ & 30,97 & & 30,00 & $\pm 75,50$ \\
\hline & E & 3,20 & 08 & 28,90 & & 53 & 28 & 6,49 & $\pm 0,06$ & 39,00 & & 170,00 & $\pm 5,5$ \\
\hline & $\mathrm{CH}$ & 1,16 & $\pm 1,10$ & 29,63 & $\pm 1,15$ & 44,67 & $\pm 2,33$ & 6,34 & $\pm 0,14$ & 106,67 & $\pm 24,96$ & 327,00 & $\pm 73,00$ \\
\hline & VA & 4,39 & $\pm 0,52$ & 28,23 & $\pm 0,45$ & 49,03 & $\pm 0,15$ & 6,50 & $\pm 0,06$ & 50,37 & $\pm 3,19$ & 197,00 & $\pm 8,00$ \\
\hline \multirow{4}{*}{ BCI } & ES & 5,73 & & 30,20 & & 56,00 & $\pm 3,41$ & 7,20 & $\pm 0,05$ & 20,67 & & 21,00 & $\pm 94,00$ \\
\hline & $\mathrm{EC}$ & 3,08 & $\pm 0,29$ & 31,63 & $-0,0$ & 47 & $\pm 0,82$ & 6,81 & $\pm 0,14$ & 38,00 & $\pm 11,75$ & 168,00 & $\pm 20,50$ \\
\hline & $\mathrm{CH}$ & 1,91 & $\pm 0,87$ & 29,43 & $\pm 0,23$ & 44,40 & $\pm 2,39$ & 7,43 & 0,17 & 126,00 & $\pm 32,25$ & 400,00 & $\pm 95,50$ \\
\hline & VA & 3,91 & $\pm 0,13$ & 28,27 & $\pm 0,81$ & 48,80 & $\pm 0,19$ & 6,95 & $\pm 0,07$ & 61,33 & $\pm 0,09$ & 247,00 & $\pm 19,00$ \\
\hline
\end{tabular}

Os resultados da ANOVA fatorial mostraram diferenças altamente significativas $(\mathrm{p}<0,01)$, entre os quatro períodos de amostragem da baía Caiçara para o oxigênio dissolvido, a transparência e a profundidade da água. Os valores médios de oxigênio dissolvido reduziram no período de cheia com o incremento da profundidade e transparência da água (Tabela 1 e Figura 2).

A temperatura da água apresentou diferenças altamente significativas entre os subsistemas da baía Caiçara (Tabela 1 e Figura 3), com os valores médios maiores nos períodos de estiagem e enchente na BCI. 
Os valores da profundidade da água estiveram positivamente correlacionados com a transparência, e seu incremento ocasionou redução concentrações de oxigênio dissolvido e da condutividade elétrica (Tabela 2).
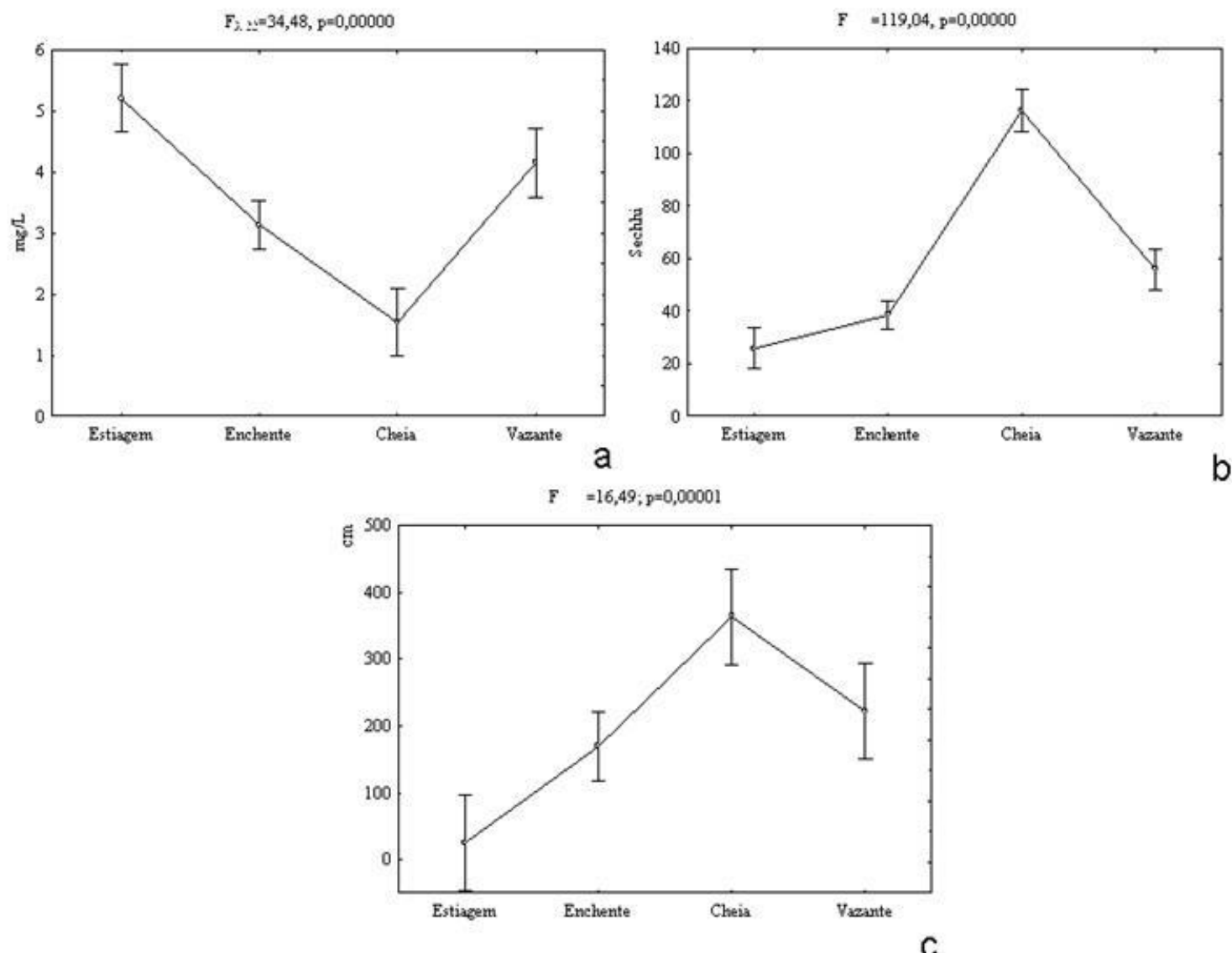

Figura 2. Valores médios das variáveis limnológicas $(n=3)$ na baía Caiçara e resultados da ANOVA fatorial para os períodos de amostragem de agosto de 2006 a maio de 2007. a - oxigênio dissolvido; b transparência; c - profundidade.

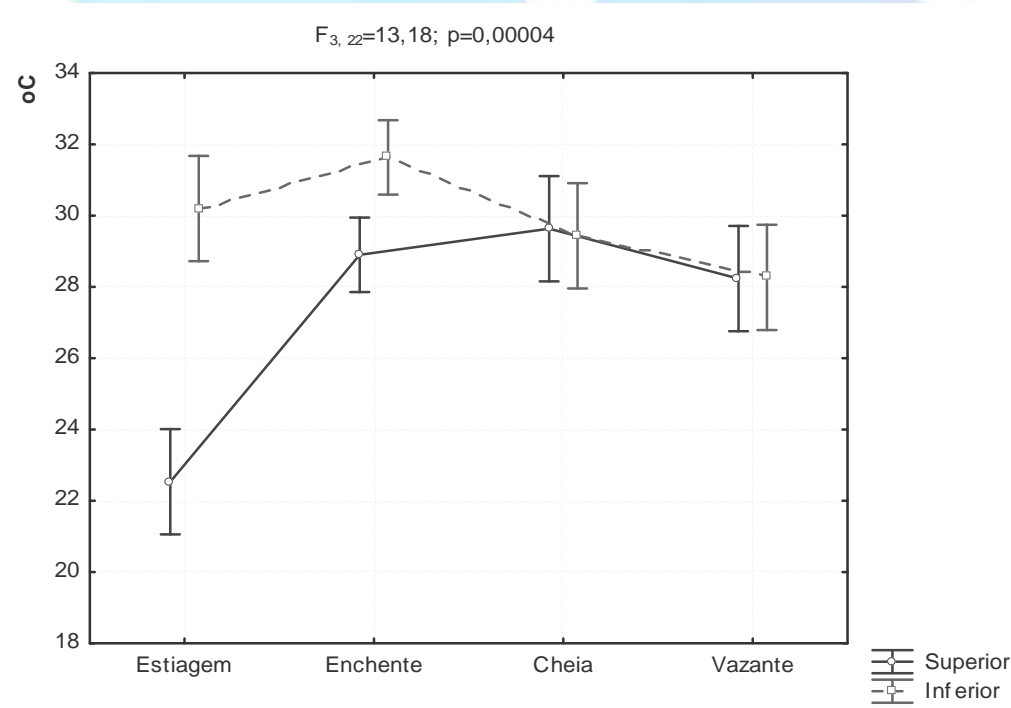

Figura 3. Valores médios da temperatura da água $(n=3)$ e resultados da ANOVA bifatorial para os subsistemas da baía Caiçara entre os períodos de amostragem de agosto de 2006 a maio de 2007. 
Tabela 2 - Índice de correlação de Spearman $\left(r_{s}\right)$ entre as variáveis limnológicas que apresentaram valores significativos ( $\mathrm{rs}>0,5)$.

\begin{tabular}{cccc}
\hline Condutividade & $\begin{array}{c}\text { Oxigênio } \\
\text { dissolvido }\end{array}$ & Condutividade & Transparência \\
\hline Transparência & 0,66 & & \\
Profundidade & $-0,57$ & & \\
& $\mathbf{- 0 , 7 5}$ & $\mathbf{- 0 , 7 3}$ & $\mathbf{0 , 7 3}$ \\
\hline
\end{tabular}

Algumas hipóteses foram levantadas para explicar a distribuição das comunidades em ambientes aquáticos e foram agrupadas como fatores biogeográficos (operando na dispersão dos organismos), e características dos habitats (tanto físico-químicas quanto bióticas, influenciando no estabelecimento e persistência da população).

No sistema de baías Caiçara foram encontrados 10.870 indivíduos associados às macrófitas aquáticas coletadas. Estes foram classificados em 4 filos, 3 subfilos, 10 classes, 2 subclasses, 20 ordens, 2 subordens, 32 famílias, 1 subfamília e 4 morfoespécies (gêneros/espécies). O Filo Arthropoda foi composto pelo maior número de táxons, sendo a Classe Insecta a mais representativa (Tabela 3 ).

Os crustáceos Copepoda (16\%) e Cladocera (11\%) foram abundantes na fauna associada e as larvas de Chironomidae (Diptera) corresponderam a 12\%. Os valores médios da abundância dos invertebrados foram maiores no período de enchente $(2.720 \pm 1.219$ ind.) e reduziram com a cheia (620 \pm 184 ind.) (Figura 4), porém sem diferenças significativas pela ANOVA fatorial.

Desta forma, pode-se perceber que, na dinâmica das águas da baía Caiçara, a profundidade foi a variável ambiental com maior número de correlações analisadas com alguns táxons (Tabela 4). Os valores de Ostracoda (Crustacea), Dytiscidae (Coleoptera), Chironomidae (Diptera) e Nematoda estiveram positivamente correlacionados com esta variável. Decapoda e Copepoda correlacionaram com os teores de oxigênio dissolvido, período favorável para estes táxons quando a profundidade esteve reduzida.

Tabela 3 - Composição espaço-temporal dos invertebrados associados à macrófitas aquáticas da baía Caiçara nos quatro períodos de amostragem entre de agosto de 2006 a maio de 2007. BCS - baía Caiçara Superior; BCI - baía Caiçara Inferior; ES - estiagem; EC - enchente; CH - cheia; VA - vazante.

\begin{tabular}{|c|c|c|c|c|c|c|c|c|}
\hline \multirow{2}{*}{$\begin{array}{l}\text { Sistemas } \\
\text { Táxons/Períodos }\end{array}$} & \multicolumn{4}{|c|}{ BCS } & \multicolumn{4}{|c|}{ BCI } \\
\hline & ES & EC & $\mathbf{C H}$ & VA & ES & EC & $\mathbf{C H}$ & VA \\
\hline Oligochaeta & 2 & 16 & 112 & 166 & 1 & 117 & 74 & 256 \\
\hline Hirudinea & & 6 & & & 2 & 1 & & \\
\hline Hydracarina & 28 & 74 & 19 & 43 & & 115 & 12 & 43 \\
\hline Eylaoidea & & & & & 3 & & & \\
\hline Hygrobatoidea & 13 & & & & 40 & 11 & & \\
\hline Araneae & 1 & & 5 & 2 & 2 & 5 & 3 & 9 \\
\hline
\end{tabular}


Crustacea

Maxillopoda

Ostracoda

Copepoda

Branchipoda

Cladocera

Conchostraca

Malacostraca

Amphipoda

Isopoda

Decapoda

Megaloptera

Neuroptera

Hymenoptera

Apidae

Ephemeroptera

Baetidae

Leptophlebiidae

Plecoptera

Odonata

Libellulidae

Trameasp.

Coenagrionidae

Trichoptera

Ochrotrichiasp.

Adicrophleps

Pyralidae

$\begin{array}{ll}50 & 162 \\ 190 & 194\end{array}$

$155 \quad 279$

$21 \quad 214$

(14

$3 \quad 7$

11

$33 \quad 6$

$1 \quad 5$

5

$1 \quad 37$

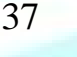

12

9

2

7

$7 \quad 6$

1

1

$21 \quad 20$

46

2

1
2

24

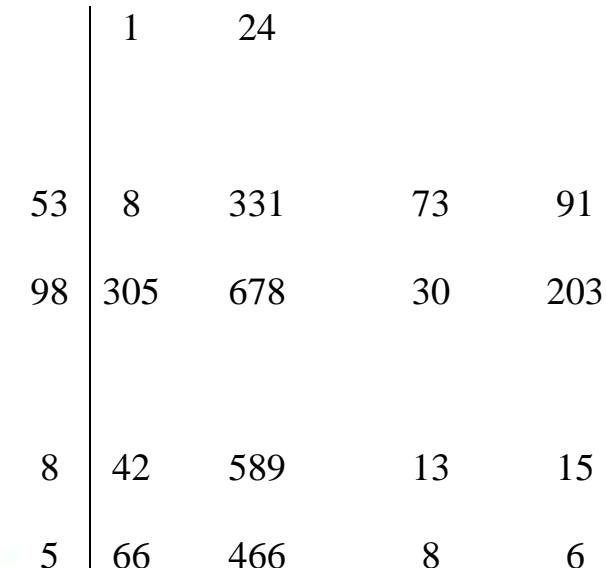

34

$5 \quad 66 \quad 466$

8

6

3

2

39

18

2

3

4
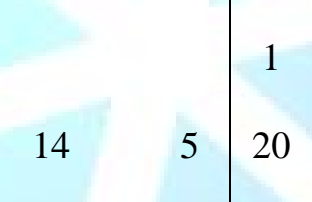

31

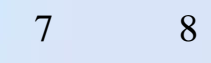

1

2

$1 \quad 7$

4

5

6

5

$5 \quad 34$

32

29

$100 \quad 40$

21

36 


\begin{tabular}{|c|c|c|c|c|c|c|c|c|}
\hline Coleoptera (adulto) & 6 & 8 & 1 & & 7 & 17 & 5 & \\
\hline Coleoptera (larva) & & 1 & 1 & & 1 & & & 1 \\
\hline Curculionidae & & 3 & & & & 3 & 1 & 7 \\
\hline Chrysomelidae & & & 1 & 1 & & & & 4 \\
\hline Elmidae & & & & & & 4 & 2 & \\
\hline Amphizoidae & 1 & & & & & & & \\
\hline Dytiscidae & & 7 & 3 & 1 & & 54 & 10 & 10 \\
\hline Gyrinidae & 30 & & 1 & & & & & 3 \\
\hline Hydrophilidae & & 2 & & & & 7 & 3 & 1 \\
\hline Hydraenidae & & 3 & & & & 7 & & \\
\hline Noteridae & 2 & 15 & 8 & 1 & & 35 & 7 & 10 \\
\hline Hemiptera & 1 & 15 & 4 & & 9 & 24 & 2 & \\
\hline Corixidae & 12 & 4 & 3 & & 6 & 18 & & 1 \\
\hline Gerridae & & & & & & & 1 & \\
\hline Hebridae & 1 & & & 2 & & & & 8 \\
\hline Vellidae & & & 4 & 4 & & 2 & & 2 \\
\hline Mesoveliidae & & 1 & & 5 & & & & 4 \\
\hline Naucoridae & & 1 & & & 5 & 3 & & 1 \\
\hline Ambrysinae & & 1 & & & & 6 & & \\
\hline Pleidae & & 10 & 2 & 28 & 1 & 17 & 5 & 10 \\
\hline Diptera & & 14 & & & & 111 & 1 & 1 \\
\hline Diptera (pupa) & 5 & 37 & 7 & 19 & 13 & 45 & 5 & 13 \\
\hline Empididae & & 1 & 1 & & & & & 1 \\
\hline Ceratopogonidae & & 53 & 17 & 54 & 19 & 173 & 39 & 128 \\
\hline Chironomidae & 12 & 70 & 183 & 281 & 46 & 132 & 122 & 448 \\
\hline Culicidae & & 76 & 4 & 52 & 1 & 81 & 7 & 79 \\
\hline Culexpipens & 4 & & & & 9 & & & \\
\hline
\end{tabular}




\begin{tabular}{|c|c|c|c|c|c|c|c|c|}
\hline Collembola & & & 3 & 5 & & 5 & & 14 \\
\hline Sminthuridae & & & & & & & & 4 \\
\hline Orthoptera & & & & & & & 1 & \\
\hline Protura & & 7 & 6 & 1 & & 95 & 7 & 1 \\
\hline Nematoda & & & & 1 & & & & \\
\hline Bryozoa (estatoblasto) & 6 & 410 & 77 & 57 & 11 & 192 & 17 & 230 \\
\hline Bryozoa (adulto) & & 61 & 10 & 1 & & 29 & 6 & 2 \\
\hline Bivalvia (Pelecypoda) & 83 & 3 & & & 40 & 2 & & 1 \\
\hline Gastropoda & 4 & 10 & 1 & 1 & 6 & 6 & & \\
\hline Prosobranchia & 5 & & & & & & & \\
\hline Planorbidae & & & 3 & 1 & & 21 & & \\
\hline Ampullaridae & & & & & 1 & & & \\
\hline Ancylidae & & & & & 1 & & & \\
\hline TOTAL & 772 & 1.858 & 750 & 937 & 820 & $\mathbf{3 . 5 8 2}$ & 490 & 1.661 \\
\hline
\end{tabular}

Tabela 4 - Índice de correlação de Spearman $\left(r_{s}\right)$ entre as variáveis bióticas e abióticas que apresentaram valores significativos ( $\mathrm{rs}>0,5)$

\begin{tabular}{lccc}
\hline & Oxigênio dissolvido & Condutividade & Profundidade \\
\hline Decapoda & 0,51 & & $-0,56$ \\
Ostracoda & & $-0,55$ & 0,60 \\
Copepoda & 0,53 & & $-0,58$ \\
Dytiscidae & & $-0,51$ & 0,50 \\
Chironomidae & & & 0,53 \\
Nematoda & $-0,65$ & $-0,57$ & $\mathbf{0 , 7 2}$ \\
\hline
\end{tabular}

Não foram observadas diferenças da abundância entre os dois subsistemas da baía Caiçara, com uma tendência de aumento na BCI (Figura 4). Como observado por Araújo (2000) e Butakka e Wantzen (2001), em sistemas pantaneiros existe relação entre as variáveis ambientais e a estrutura da comunidade de invertebrados aquáticos, o que pode ser um fator determinante para a compreensão de determinados grupos. 


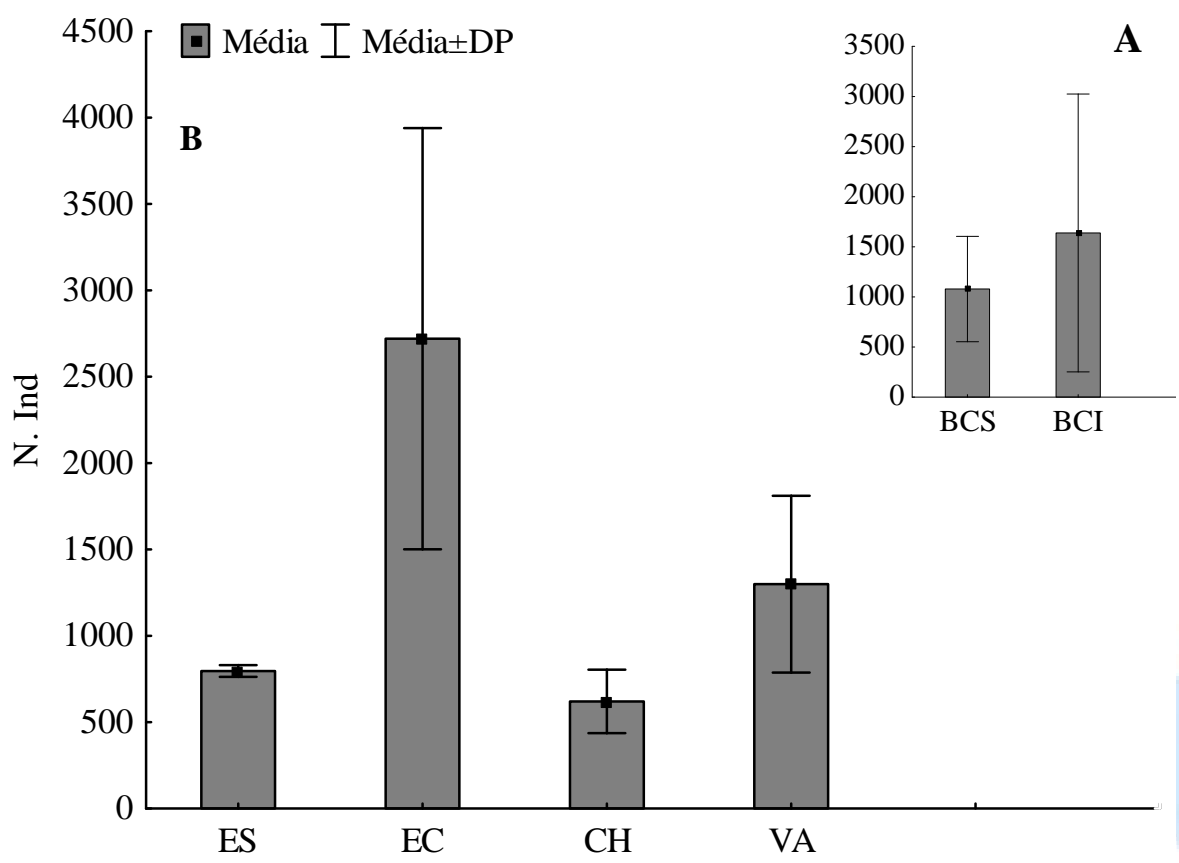

Figura 4. Valores da abundância média (A) de invertebrados associados às macrófitas aquáticas nos sistemas da baía Caiçara Superior (BCS) e baía Caiçara Inferior (BCI), e (B) nos períodos de amostragem, entre de agosto de 2006 a maio de 2007.

Neste estudo pode-se inferir que os grupos mais influenciados pela dinâmica do pulso de inundação foram os microcrustáceos (correlacionados com os teores de oxigênio dissolvido), os coleópteros da família Dytiscidae, os dípteros da família Chironomidaee os nemátodos (correlacionados com a profundidade da água), decompositores da matéria orgânica.

No período de cheia, a lixiviação da serapilheira da porção terrestre eleva o material orgânico dentro do sistema, além da senescência de boa parte das macrófitas aquáticas, incrementando alguns desses decompositores nas baías.

A profundidade da coluna da água, ou seja a amplitude do pulso de inundação, foi um dos fatores ambientais mais importantes para a dinâmica destes grupos de invertebrados dentro das comunidades. O tamanho do ecossistema em um determinado período é uma peculiaridade importante que pode afetar direta e indiretamente mecanismos que determinam os padrões dos atributos dos táxons em escalas locais (SCHEFFER et al., 2006). O fator de diluição faz com que os grupos taxonômicos se dispersem mais pela planície, contendo maior área para procurar refúgio e alimento.

Os microcrustáceos, organismos determinantes na análise da sazonalidade (HWANG et al., 2005), competem pela matéria fina particulada (SIMÕES et al., 2011). Para esses pesquisadores, esses grupos podem ser influenciados tanto pelas características físico-químicas quanto pela predação e presença de macrofitas aquáticas.

Os coleópteros e dípteros são grupos generalistas e sua relação positiva com a profundidade denota que algumas espécies apresentam resistência e resiliência frente às mudanças ambientais. 


\section{CONCLUSÕES}

Esta pesquisa demonstrou que as variáveis analisadas influenciaram de forma diferente para os níveis taxonômicos identificados na composição e abundância de invertebrados associados às macrófitas aquáticas.

Pode-se observar a relação entre as variáveis limnológicas e os invertebrados presentes nos estandes de macrófitas. A amplitude do pulso de inundação e o oxigênio dissolvido foram as principais variáveis que determinaram a composição e a abundância de grupos taxonômicos no sistema da baía Caiçara.

Estudos como estes são importantes para que haja o manejo e conservação adequados no Pantanal, tendo em vista a importância dos invertebrados aquáticos na bioindicação de ecossistemas aquáticos.

\section{REFERÊNCIAS}

ARAÚJO, A.A. Caracterização limnológica e a comunidade de macroinvertebrados bentônicos do "Rio Claro Rio Pixaim", Pantanal MatoGrossense, Poconé-MT. 2000. 76 f. Tese (Doutorado) - Instituto de Biociências, Universidade Federal de Mato Grosso, Cuiabá, 2000.

BUTAKKA, C. M. M.; WANTZEN, K.M. Correlações entre variações ambientais e distribuição de larvas de Campsurus sp. (Ephemeroptera-Polymitarcydae) na baía de Sinhá Mariana, Pantanal Mato-Grossense, MT. In: SIMPÓSIO SOBRE RECURSOS NATURAIS E SÓCIOECONÔMICOS DO PANTANAL, 3., 2000, Corumbá. Anais... Corumbá: Embrapa Pantanal, 2001. p. 1-17.

COFFMAN, W.P.; FERRINGTON JR., L.C. Chironomidae. In: MERRIT, R. W.; K. W. Cummins (Eds.) An introduction to the aquatic insects of North America. Dubuque: Kendall/Hunt Publishing Company. 1996. p. 635-643.

DA SILVA, C.J. Nota previa sobre o significado biológico dos termos usados no Pantanal Matogrossense. I - "Batume” e "Diquada". Revista da UFMT, v. 4, p. 30-36, 1984.

DA SILVA, C.J; PINTO-SILVA, V. Macrófitas aquáticas e as condições físicas e químicas dos "alagados", "corixos" e rios, ao longo da rodovia Transpantaneira - Pantanal Matogrossense (Poconé-MT). Revista Brasileira de Biologia, v. 49, n. 3; p. 691-697, 1989.

ESTEVES, F.A. Fundamentos de limnologia. Rio de Janeiro: Interciência, 1998. 602 p.

FERREIRA, W.R.; PAIVA, L.T.; CALLISTO, M. Development of a benthic multimetric índex for biomonitoring of a neotropical watershed. Brazilian Journal of Biology, v. 71, n. 1, p. 15-25, 2011.

GOULART, M.D.C.; CALLISTO, M. Bioindicadores de qualidade de água como ferramenta em estudos de impacto ambiental. Revista da FAPAM, Pará de Minas, v. 2, n. 2, p. 153-164, 2003.

HARRIS, M.B.; TOMAS, W.M.; MOURÃO. G.; DA SILVA, C.J.; GUIMARÃES, E.; SONODA, F.; FACHIM, E. Desafios para proteger o Pantanal brasileiro: ameaças e iniciativas em conservação. Megadiversidade, Belo Horizonte, v. 1, n. 1, p. 156-164, 2005. 
HWANG, D.; RUST; A.G.; RAMSEY, S.; SMITH, J.J.; LESLIE, D.M.; WESTON, A.D.; DE ATAURI, P.; AITCHISON J.D.; HOOD, L.; SIEGEL, A.F.; BOLOURI, H. A data integration methodology for systems biology. Proceedings of the National Academy of Sciences of the United States of America, Melville, v. 102, n. 48, p. 17296-17301, 2005.

JUNK, W.J.; DA SILVA, C.J. O conceito do pulso de inundação e suas implicações para o Pantanal de Mato Grosso. In: SIMPÓSIO SOBRE RECURSOS NATURAIS E SÓCIO-ECONÔMICOS DO PANTANAL. 2., 1996. Anais... Corumbá: Embrapa, 1999. p. 17-28.

MERRITT, R.W.; CUMMINS, K.W.; BERG, M.B. (Eds.). An introduction to the aquatic insects of North America. Dubuque: Kendall/Hunt Publishing Company, 2008. 1158 p.

MUNIZ, C.C. Composição da comunidade íctica em área limnética, relacionada ao ciclo hidrológico nas Baías da Salobra e Negra, no Pantanal de Cáceres - MT. 2005. 71 f. Dissertação (Mestrado) - Universidade Federal de Mato Grosso, Cuiabá, 2005.

RUSSO, M.R.; FERREIRA, A.; DIAS, R.M. Disponibilidade de invertebrados aquáticos para peixes bentófagos de dois riachos da bacia do rio Iguaçu, Estado do Paraná, Brasil. Acta Scientiarum, Maringá, v. 24, n. 2, p. 411-417, 2002.

SCHEFFER, M.; VAN GEEST, G.J.; ZIMMER, K.; JEPPESEN, E.; SØNDERGAARD, M.; BUTLER, M.G.; HANSON, M.A.; DECLERCK, S.; DE MEESTER, L. Small habitat size and isolation can promote species richness: second-order effects on biodiversity in shallow lakes and ponds. Oikos, Rio de Janeiro, v. 112, n. 1, p. 227-231, 2006.

SIMÕES, N.R.; RIBEIRO, S.M.M.S.; SONODA, S.L. Diversity and structure of microcrustacean assemblages (Cladocera and Copepoda) and limnological variability in perennial and intermittent pools in a semi-arid region, Bahia, Brazil. Iheringia. Série Zoologia, Porto Alegre, v. 101, n. 4, p. 317-324, 2011.

ZILLMER, T.A.; VARELLA, R.F.; ROSSETE, A.N. Avaliação de algumas características físicoquímicas da água do Ribeirão Salgadinho, Nova Xavantina-MT. Holos Environment, Rio Claro, v. 7, n. 2, p. 123-138, 2007. 